\title{
RHINOSPORIDIUM SEEBERI EN IRAN
}

\section{Par M. HABIBI}

Nous avons présenté, en mars 1939, les trois premiers cas de Rhinosporidium seeberi observé en Iran, dans les Annales de Parasitologie humaine et comparée. Depuis, nous avons rencontré 28 autres cas dont nous présentons ici la statistique. Cette affection s'est présentée chez nos malades dans l'œil et dans le nez sous forme de polypes, richement vascularisés, de grosseur variable, de consistance molle, friable, de couleur rouge, surface irrégulière et bosselée.

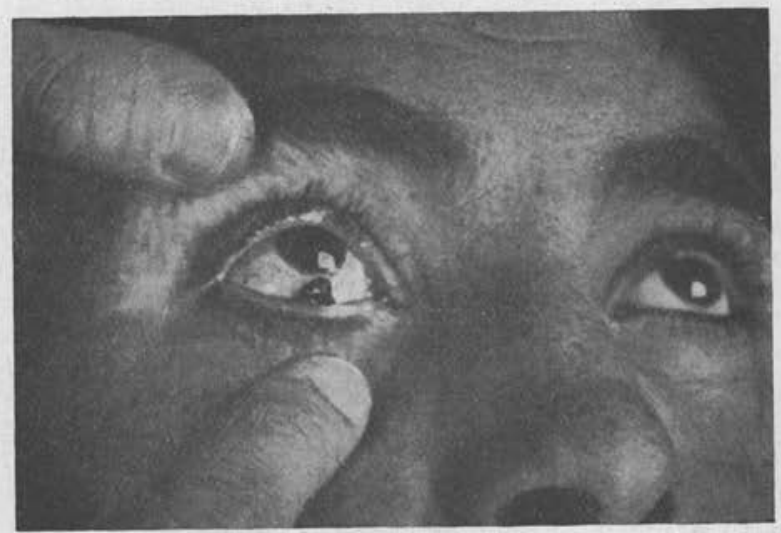

FIg. 1. - Polype à Rhinosporidium seeberi, observation $\mathrm{n}^{\circ} 30$.

Cette maladie est très rare. Le nombre d'observations jusqu'en 1938 ne dépasse pas 165 dans le monde entier. On la rencontre à Ceylan, en Amérique, en Afrique, et en Iran, notamment à Téhéran et Ardébile.

Jusqu'en 1937, l'existence de cette affection était inconnue en Iran. Depuis, nous avons eu l'occasion d'observer 31 cas. Le nombre des malades est sûrement beaucoup plus élevé, puisqu'en Iran l'examen histologique des tumeurs prélevées du nez et de l'œil est loin d’être généralisé.

Ann. de Parasitologie, T. XXII, nos 1-2, 1947, p. 84-88. 
ObSERvation $\mathrm{N}^{\circ} 30$. - H..., garçon de 17 ans, originaire d'Ardébile, garçon de café. Consulte la clinique d'opthalmologie pour une petite tumeur polypeuse, rougeâtre, de la paupière inférieure de l'œil gauche, apparue pour la première fois il $\mathrm{y}$ a deux ans.

A l'examen clinique on trouve une tumeur pédiculée, implantée sur la face interne de la paupière, d'une couleur rouge foncée, surface irrégulière, saignant facilement au toucher, dépassant $3 \mathrm{~mm}$. du bord de la paupière.

Pas de lésion de la fosse nasale de l'œil droit.

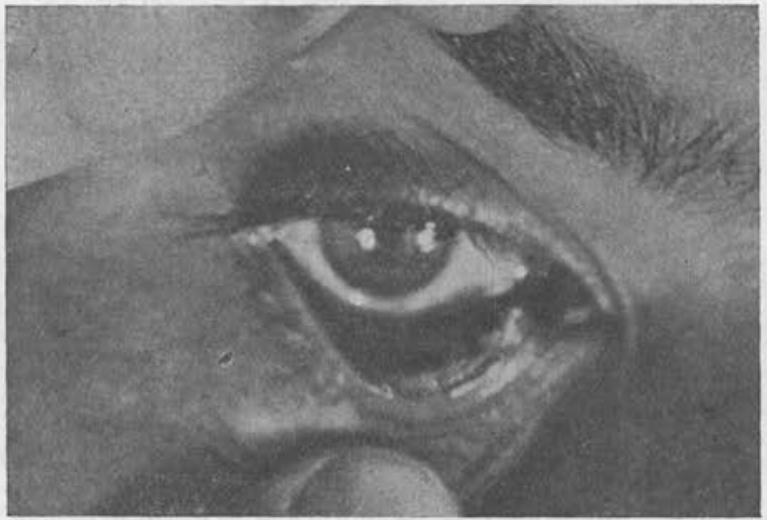

Fı, 2. - Polype à Rhinosporidium seeberi, observation $\mathrm{n}^{\circ} 30$.

La tumeur était enlevée il y a 1 an par un coup de ciseaux en faisant la cautérisation de sa base; pendant 1 an, pas de récidive, mais depuis quelques mois la tumeur s'est développée de nouveau.

La tumeur enlevée a présenté histologiquement la structure d'un polype causé par le Rhinosporidium.

Observation $\mathrm{N}^{\circ}$ 31. - D..., garçon de 19 ans, écolier, originaire d'Ardébile.

Consulte la clinique d'ophthalmologie pour une tumeur polypeuse, de couleur rouge, irrégulière, de l'angle externe de l'œil gauche, apparue pour la première fois il y a 5 ans.

Depuis, le malade, en faisant un nœud avec un crin ou de la soie autour du pédicule de la tumeur, a fait disparaitre sa tumeur, mais quelques mois après la tumeur a récidivé. (Il paraît que ce genre de traitement est bien connu parmi les paysans d'Ardébile car cette maladie est très répandue dans cette région). 
Pas de lésion dans les fosses nasales et dans l'autre œil.

La tumeur enlevée a présenté histologiquement la structure d'un polype causé par le rhinosporidium.

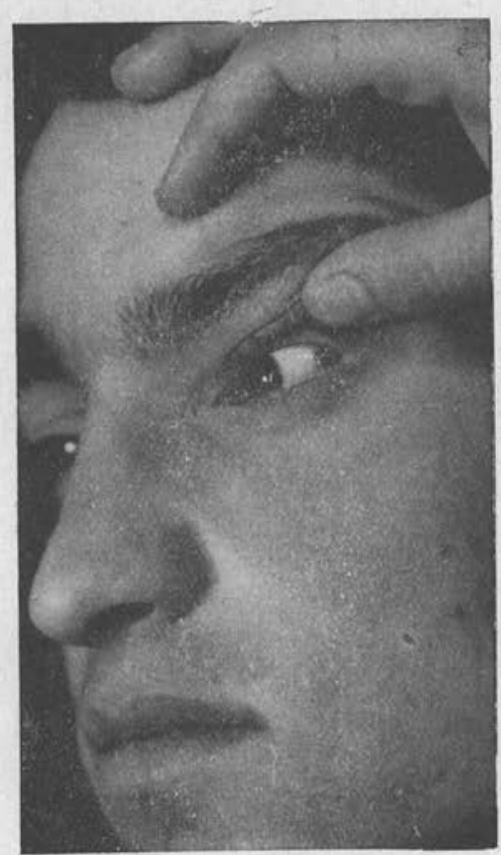

Fig. 3. - Polype à Rhinosporidium seeberi, observation $\mathrm{n}^{\circ} 31$.

L'étude clinique de nos malades a donné les résultats suivants :

$1^{\circ}$ Cette affection se rencontre à tout âge, néanmoins elle est plus fréquente entre 10 et 30 ans :

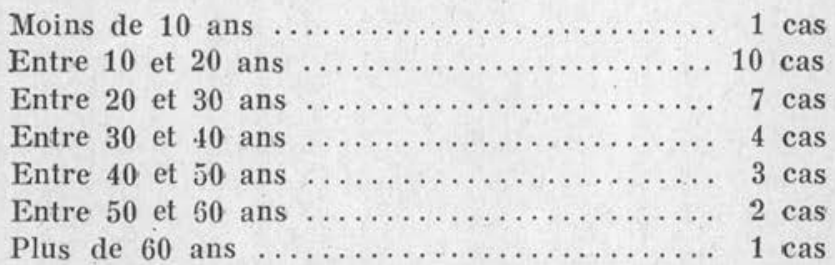

$2^{\circ}$ L'influence du métier aussi ne joue aucun rôle :

9 de nos malades étaient écoliers.

7 de nos malades étaient ouvriers. 
6 de nos malades étaient commerçants.

5 de nos malades étaient sans occupation (femmes).

4 de nos malades étaient fonctionnaires.

D'après cette statistique, évidemment bien restreinte, cette affection est fréquente parmi les écoliers, ce qui montre que la contagion directe doit jouer un rôle assez important.

$3^{\circ}$ Suivant la distribution géographique :

16 venaient d'Ardebile.

13 venaient de Téhéran.

1 venait d'Ispahan.

1 venait de Kermanchah.

Sur ce nombre, 7 malades étaient des femmes.

$4^{\circ}$ Du point de vue de la localisation, on a trouvé :

22 polypes du nez.

7 polypes de l'œil et des paupières.

2 polypes multiples de la fosse nasale et de l'œil.

A l'examen microscopique, les parasites se présentent dans les tissus sous forme de masses arrondies, variant entre 6 et $300 \mu$ de diamètre.

Le sporange contient des milliers de spores qui sont mises en liberté par un pore périphérique qui se creuse en un point déterminé de la membrane du parasite. Si cette ouverture n'est pas assez large, et si la pression intérieure est très forte, les spores sont rejetées violemment en dehors du sporange de façon à faire retourner la membrane périphérique sur elle-même.

L'étude histologique des tumeurs à Rhinosporidium se caractérise tout d'abord par la présence du parasite dans le tissu tumoral. La réaction tissulaire consiste en une réaction inflammatoire à corps étrangers. On y distingue :

a) une réaction macrophagique intense, accompagnée d'une sclérose plus ou moins marquée, d'une infiltration lympho-plasmocytaire diffuse et des cellules lipophages et éosinophiles ;

b) des lésions folliculaires, type tuberculoïde et sporotrichosique.

L'inoculation expérimentale chez l'homme dans l'œil, et chez les animaux, notamment le cobaye, le lapin, le mouton et le bœuf, dans l'œil, le nez et sous la peau, est restée sans succès (Ch. Oberling, L. Delpy, M. Habibi, 1941).

Pour le traitement, Wright a proposé l'instillation d'une solution 
d'émétique à $20 / 0$, mais nous n'en avons tiré aucun résultat satisfaisant. Chams et Bastan ont essayé, avec de bons résultats, l'électrocoagulation de la tumeur et des régions avoisinantes. Max Mever, $\mathrm{H}$. Adle et $\mathrm{Dj}$. Aalam ont essayé l'extirpation chirurgicale avec cautérisation de la base de la tumeur. Le résultat était satisfaisant.

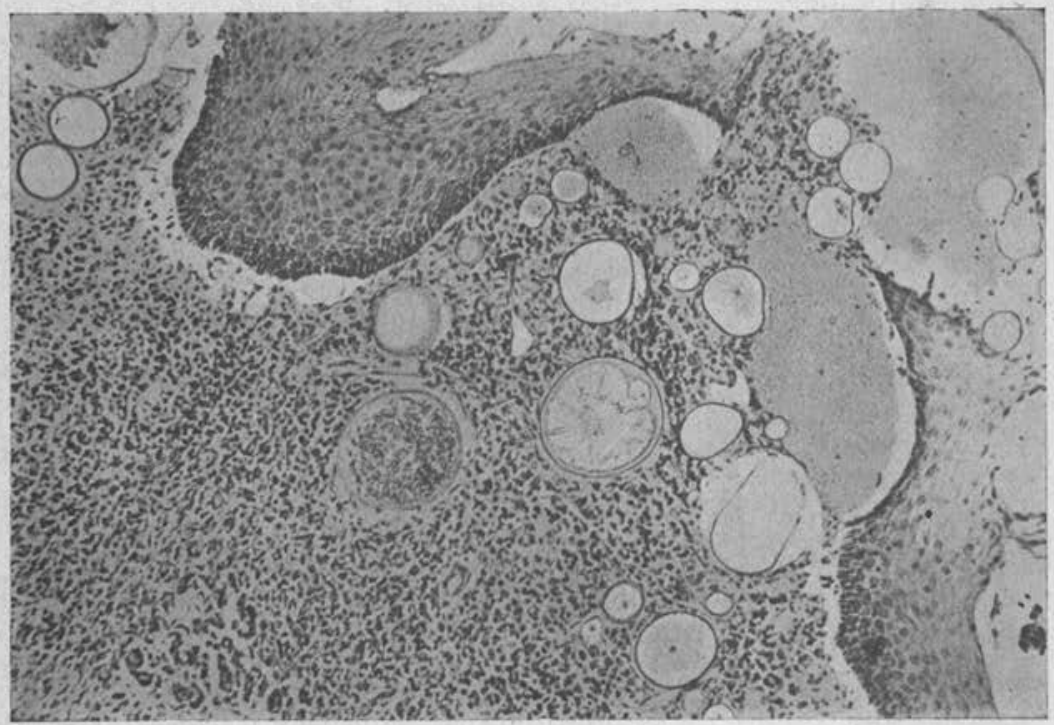

Fig. 4. - Structure d'un polype à Rhinosporidium seeberi.

\section{Bibliographie}

HABIBI (M.). - Contribution à l'étude de Rhinosporidium seeberi. Description de 16 cas observés en Iran. Pratica oto-rhino-laryngologica, Revue internationale d'otolaryngologie, VI, 1944 , p. 35.

- Etude de 3 cas de Rhinosporidium seeberi en Iran. Annales de Parasitologie humaine et comparée, XVII, 1939, p. .

- Revue de la Science Médicale Française du Moyen-Orient, $\mathrm{n}^{\circ} 10$, juillet 1943.

Max Meyer. - Bluten der Septumpolype durch Rhinosporidium seeberi. V, Revue internat. d'otolaryngol., 1944, p. 139.

Laboratoire d'Anatomie pathologique de la Faculté de médecine de Téhéran 